\title{
ZUR MIKROSKOPISCHEN ANATOMIE DES BLUTGEFÄSSYSTEMS DER SKORPIONEN.
}

\author{
VON \\ Dr. E. N. PAWLOWSKY
}

Professor der Zoologie an der Militär-Medizinischen Akademie zu Petrograd.

Mit I Tafel.

Die anatomische Untersuchung des Blutgefässystems gehört eigentlich nicht zu den Aufgaben der vorliegenden Arbeit. Diesbezügliche Angaben finden sich bei Newport (I843), Dufour (1856), Blanchard (I851-i859), Lankester ( 1885 ), Houssay ( 1886,1887 ), Bernard und Petzunkevitch (I922). Ich werde nur mit grösserer Ausführlichkeit auf die Betrachtung des mikroskopischen Baues des Herzens und der Blutgefässe des Skorpions eingehen. Das $z \mathfrak{u}$ schildernde System der Organe, namentlich das Blutgefässsystem, welches sowohl durch Arterien als auch durch Venensinusse vertreten ist, ist sehr gut entwickelt. Ich werde die Schilderung mit dem Herzen beginnen, welches in Form eines zylindrischen Schlauches auf der Leber im Plaeabdomen gelegen ist. Gadsikiewicz (I908) schildert den Bau dieses Organs beim Euscorpius folgendermassen.

Das Herz ist dreischichtig; die äussere Schicht besteht aus longitudinalen Bindegewebefasern und glatten Muskelfasern, welchen Perikardzellen anliegen. Die mittlere Schicht ist ,aus zwei Reihen in bogenförmiger Anordnung gelegener Muskelfasern gebildet, die zur rechten und linken Seite des Herzens liegen und sich miteinander in der ventralen und dorsalen Mittellinie verbinden" (1. c., S. 4). Die Fasern des atypischen quergestreiften Muskelgewebes sind von einem Sarkolemm überzogen, das sich innerhalb des Herzens zu einer homogenen Intima verdickt, deren freie Oberfläche mit einem aus Endothelzeilen bestehenden Endokard bedeckt ist. Ein mesodermales Endokard sahen im Herzen des Skorpions Kowalewski und Schulgin (I886); Schneider (i892) konnte sogar die Anwesenheit desselben durch Imprägnierung der Zellgrenzen mit salpetersaurem Silber nachweisen.

In das Herz führen Ostien, deren Ränder mit nach innen hinabhängenden 
Klappen versehen sind. Die letzten bestehen nur aus einer zirkulären Muskelfasernschicht. Besondere Kammern gibt es im Herzen, nach Gadsikiewicz, nicht. Die arteriellen Klappen sind ebenfalls auf Kosten der Ringsmuskulatur gebildet.

Das von Gadsikiewicz entworfene Schema der Herzstruktur entspricht im allgemeinen der Wirklichkeit, nur kann man nicht seiner Deutung mancher Struktureinzelheiten beipflichten. Es handelt sich um das Sarkolemm und das Herzendothel. „Jedes Fibrillenbündel ist von allen Seiten von Sarkolemm umgeben" (Figg. 4, 6 sar) (1. c., S. 5). Das Sarkolemm ist oft von den Fibrillen durch einen leeren Raum getrennt, dessen Entstehung GadsikiEwicz darauf zurückführt, dass während der Diastole die Fasern sich vom Sarkolemm ablösen (!). Schon in den nächsten Zeilen bezweifelt der genannte Autor, ob das von ihm geschilderte Gebilde auch in der Tat echtes Sarkolemm sei, da es sich rot nach van Gieson färbt. Kerne sind in demselben nicht gefunden worden. Die Anwesenheit der Kerne würde dafür sprechen, dass diese Hülle kein Sarkolemm sei. Auf diese Weise ist diese Frage in der Hauptsache offen geblieben, obwohl GadsıKIEwicz in seiner Arbeit wiederholt die Bezeichnung Sarkolemm gebraucht.

Es genügt jedoch, auf die Figg. 3, 5, 6, 7 der Gadsikiewicz'schen Arbeit einen Blick zu werfen, um sich zu überzeugen, dass die Präparate vom Verfasser falsch gedeutet werden. Die Muskelfasern der abgebildeten Objekte schrumpiten beim Fixieren und Einbetten zusammen, weshalb zwischen ihnen weite Lücken zurückblieben. Das, was Gadsıkıewicz für Sarkolemm hält, ist in der Tat ein bindegewebiges plattenähnliches Gebilde, welches von allen Seiten die Herzmuskelfasern umkleidet, d. h. das angebliche Sarkolemm ist in der Tat Perimysium oder interstitielles Bindegewebe. Es wird nun begreiflich, weshalb das "Sarkolemm" von Gadsikiewicz sich wie Bindegewebe färbte. Die Muskelfasern sind ausserdem von einem echten Sarkolemm umkleidet, welches eine sehr dünne Hülle darstellt, eng die Muskelfasern umschliesst und sämtlichen Formveränderungen derselben während ihrer Kontraktion folgt. Was nun den Ursprung des Sarkolemms beim Skorpion betrifft, ob es ein Produkt des umgebenden Bindegewebes oder ein solches des Sarkoplasmas der Muskelfasern ist - kann ich nicht entscheiden. Diese Frage ist auch in bezug auf die Säugetiere in endgültiger Form noch nicht gelöst. Jedenfalls ist das Vorhandensein von Sarkolemm in dem Herzmuskel des Skorpions über jeden $Z$ weifel erhaben.

Von innen ist das Herz mit einer Hülle ausgekleidet, welche abgeplattete chromatinreiche Kerne besitzt (Taf. I, Figg. I, 4 end). Kerne gibt es auch im Perimysium, welches zwischen den Muskelfasern gelegen ist, und ich kann nicht begreifen, weshalb Gadsikiewicz sie in seinen Präparaten nicht finden konnte. Das Perimysium der äusseren Herzoberfläche geht in Bindegewebe, welches die äusseren I.ängsfasern des Herzens umgibt und in platten- 
ähnliches faseriges Gewebe, in dessen Lücken ovale Perikardzellen gelegen sind, über. Die Fasern der äusseren Herzhülle sind tatsächlich glatt; es gelang nie, selbst Spuren einer Querstreifung in ihnen festzustellen. GadSIKIEWICZ bezeichnete sie als glatte Muskelfasern. Es ist möglich, dass sie zum Muskelgewebe gehören. Auf jeden Fall stellen diese Fasern vollständig entwickelte histologische Elemente dar. Sie sind ziemlich dick (Taf. I, Fig. 5 ecrf), sind von einem eigenen interstitiellen Bindegewebe umkleidet und unterscheiden sich vom Bindegewebe dadurch, dass ihnen die Fähigkeit, sich nach MaLlory blau zu färben, fehlt. Eisenhämatoxylin halten sie sehr fest. Indem sie sich in ihrem Verhalten zum MALLORY'schen Farbstoff von dem des Bindegewebes unterscheiden, weisen sie auch Unterschiede gegenüber den Muskelfasern auf, welche sich nach MANN-Holi.A'de himbeerrot färben.

Was stellt nun das Herzendothel beim Skorpion dar? Diese Frage kann man an Hand von Präparaten lösen, an welchen sich stellenweise die innere Herzhïlle bei der Bearbeitung der Objekte ablöste. Einer derartigen Erscheinung begegnete nicht selten auch GaDsikiewicz, welcher annimmt, dass in solchen Fällen pathologische Prozesse stattfinden mögen, die eine Veränderung auch seitens des Endothels herbeiführen. Die Zellen des letzteren werden dabei grösser und deutlicher (1908, Fig. 7 end, $z$ ).

Bei der Betrachtung der abgelösten inneren Herzhülle des Skorpions kann man sich überzeugen, dass dieselbe mehrschichtig sei. Die Ablösung kann verschiedenartig ausfallen. In einem Falle erhebt sich über einigen Ringsfasern die innere Schicht der Herzhülle, welche abgeplattete Kerne besitzt, in Form einer Blase. Die übrigen Schichten, welche ebenfalls Kerne besitzen, bleiben an den Muskelfasern zurück. An dem Längsschnitte des Herzens ist der Übergang der Hülle von der Vorwölbung (vorragenden Convexität) einer Faser zur anderen deutlich zu sehen, sowie auch das Eindringen der kernhaltigen Zwischensubstanz in die Spalträume zwischen den Fasern (Endomysium). In solchen Fällen fand eine Spaltung der inneren Herzhülle statt, so dass ein Teil der Schichten mit dem Sarkolemm über den Muskelfasern zurückblieb (Taf. I, Fig. I end). An anderen Stellen desselben Präparates treten andere Verhältnisse hervor. Unter der sich erhebenden Blase der inneren Herzhülle liegen Muskelfasern mit einer rauhen, gleichsam zerrissenen Oberfläche. Diese Beschaffenheit der Oberfläche hängt davon $a b$, dass sich beim Ablösen der Herzhülle von den Muskelfasern auch das Sarkolemm mit ablöste, welches eng mit dem Sarkoplasma verbunden ist, so dass sich beim Ablösen das Sarkolemm von dem unterliegenden Muskelgewebe losreisst und somit der nunmehr entblössten Muskelfaser eine rauhe Oberfläche verleiht. Eine genaue Betrachtung derartiger Bilder und ein Vergleich derselben mit normalen Stellen derselben Präparate zeigen deutlich, dass das Endothel des Skorpionherzens ein kompliziertes Gebilde sei, dessen Hauptmasse aus dem Bindegewebe des Perimysiums besteht. Ist ein Endothel an 
der inneren Fläche des Perimysiums vorhanden? Ich habe keine Imprägnierungsversuche an den Skorpionherzen vorgenommen und ist mir leider auch die Schneiner'sche Arbeit im Original unbekannt gebiieben. Wie dem auch sei, neige ich durchaus zur negativen Lösung der gestellten Frage, weil die innere Auskleidung des Skorpionherzens um die Klappenränder herum unmittelbar in das Perimysium der Aussenfläche übergeht; das letzte aber besitzt keinerlei Elemente endothelialer Natur.

Die Struktur des Skorpionherzens stelle ich mir folgendermassen vor. Das Stroma des Organs ist aus Bindegewebe gebildet; dasselbe ist räumlich mit Fasern, an welchen das Herz aufgehängt ist, mit plattenähnlichen Nephrozyten-tragenden Gebilden, mit venoperikardiale Muskeln bekleidenden Hüllen usw. verbunden. In diesem Bindegewebe (des Stromas), welches seitens der Herzhöhle scharf konturiert ist, ist eine Schicht halbkreisförmiger quergestreifter Muskelfasern gelegen, welche die Hauptmasse der Herzwand darstellt (Taf. I, Figg. I, 2, $4 \mathrm{em}$ ); ausserhalb dieser Schicht liegen in demselben Stroma longitudinale Fasern, welchen die Querstreifung fehlt (Taf. I, Figg. I, 2 ecr). Das bindegewebige Stroma stellt Endo- und Perimysium dar, welches seitens der Herzhöhle in mehreren Schichten aufeinanderlagert. Es lässt sich nicht nachweisen, dass die innere Schicht eben Endothel und nicht einfach eine Bindegewebeschicht sei, welche der inneren Auskleidung der Arterien und Sinusse analog ist. Demnach haben wir im Herzen des Skorpions eigentlich nur zwei Schichten - eine zirkuläre Muskelschicht (Myokard) und eine longitudinale äussere faserige Schicht (Ektokard). Die Intima (Endokard) wird durch das Perimysium dargestellt, welches von innen die Fasern des Herzens überzieht und mit dem Endomysium, das wiederum in die bindegewebige äussere Herzhülle übergeht, verbunden ist. Daher kann man die Intima nur mit Vorbehalt für eine selbständige Herzhülle halten. $\mathrm{Ob}$ dieselbe an ihrer freien Oberfläche eine homogene membrana propria absondert, wie sie Livanoff und Bouroff (1916) bei den Blutgefässen der Blutegel schilderten, kann ich nicht mit Sicherheit behaupten. Die Intima des Skorpionherzens entspricht vollständig der bindegewebigen Auskleidung der Blutgefässe bei den Ringelwürmern (Anneliden, Lrvanoff I9I3, Selensky I9I5).

Hinsichtlich der Struktur der quergestreiften Herzmuskulatur kann nichts Besonderes hinzugefügt werden. Die Untersuchung des Charakters der Querstreifung der Fasern muss zum Gegenstand einer speziellen Arbeit werden. was den Rahmen meiner Aufgabe überschreiten würde. In den Muskelfasern des Herzens ist viel Sarkoplasma vorhanden; entlang der Achse der Muskelelemente sind gestreckte Kerne gelegen. In das Sarkoplasma ist eine grosse Anzahl Muskelfibrillen versenkt, die sich spiralförmig um die Längsachse der Faser winden. Häufig, dasselbe hat auch Gadsikiewicz bemerkt, divergieren die Muskelfibrillen auf einer gewissen Ausdehnung; der 


\section{ANATOMIE DES BLUTGEFÄSSYSTEMS DER SKORPIONEN ${ }^{465}$}

zwischen ihnen entstandene Raum ist von einem feinkörnigen Stoff ausgefüllt. Es ist möglich, dass manche von derartigen Gebilden intravital entstehen.

Die Querstreifung der Fibrillen ist weniger sichtbar, als das an den willkürlichen Muskeln der Fall ist; auch die Art der Querstreifung der Fibrillen ist von der der letzten verschieden. GadsıkıEwicz weist darauf hin, dass das Sarkolemm sich an der Entstehung der äusseren Streifung der Faser beteiligt, wobei die Querbänder desselben (des Sarkolemms) im Gegensatz zu den Myofibrillen mit Eisenhämatoxylin nicht färbbar seien. Schon dieser letzte Umstand spricht in ganz bestimmter Weise gegen die Spezifität derjenigen Querbänder, die Gadsikiewicz an dem "Sarkolemm” der Muskelfasern beobachtete, da das HeIdenhein'sche Eisenhämatoxylin als Reagens für Querstreifung gilt. Ich erwähnte bereits oben, dass GaDsikiewicz das Perimysium für Sarkolemm annahm; das Perimysium vermag in der Tat Querfalten (beim Fixieren?) zu bilden, welche aber keine Beziehung zu der spezifischen Streifung der eigentlichen Muskelfaser haben. Der Hinweis Gadsikiewicz's auf die Untersuchungen von ENDERLEIN (I900), laut welchen das Sarkolemm bei den Insekten an der Querstreifung partizipiert, ist nicht beweiskräftig, da bei ENDERLEIN die Rede vom Sarkolenmm ist, während Gadsikinwicz fälschlich das Perimysium für Sarkolemm anspricht. Der letztgenannte Autor bemerkt im übrigen, dass das „Sarkolemm” (in seinem Sinne) des Skorpions sich an der Bildung des Krause'schen Diskus nicht beteilige, während bei den Insekten das Gegenteil der Fall ist. Das echte Sarkolemm ist zweifelsohne in die Bildung der Querstreifung verwickelt, da an seiner Innenfläche die Inofragmen-Diskus $Z$ und $M$ befestigt sind. Dieselben stellen Membranen dar, welche „die ganze Muskelfaser in der Querrichtung in einzelne Scheiben schneiden und selbst in der Längsrichtung von Fibrillen durchbohrt werden" (Maximow 1915, S. 319).

Die Schicht der zirkulären Muskelfasern entspricht, scheinbar, der „tunique interne ou propre”, die Dufoun im Skorpionherzen gesehen hat. Dieselbe besteht aus „fibres spiroïdes”, welche der Autor auf Fig. 9, Taf. I seiner im Jahre 1856 erschienenen Arbeit abbildet. Mir gelang es ebenfalls, das Herz beim Präparieren zu einem spiralförmigen Bande auszuziehen, wobei die Ostien stets die Mitte des Bandes einnahmen und nicht auseinanderrissen. Ein derartiges Auseinanderwickeln des Herzens scheint wohl artifiziell zu sein. Man kann nicht behaupten, dass das Herz aus einem spiralförmig gewundenen Bande besteht, da in die Breite der Spirale sich eine grosse Anzahl zirkulärer Muskelfasern legen. Die letzten, wie es GadsıkıEwicz beobachtete, vereinen sich in der Medianlinie des Herzens in der Weise, dass jede Faser einer Seite mit zwei Fasern der entgegengesetzten verbunden wird. An der Verbindungsstelle ist ein stark lichtbrechender Streifen zu sehen, welcher sich nach MALLORY blau färbt. Eine derartige Verbindungsweise macht eben das Ausziehen des Herzens zu einem spiralen Bande möglich. 
Die Herzostienklappen sind durch GadsiKIEwICz richtig beschrieben worden. Ich kann nur hinzufügen, dass an dem Bau ihrer Muskelschicht zwei Reihen von Ringmuskelfasern teilnehmen (Taf. I, Fig. 2), nicht eine, wie das im Herzen der Fall ist. An dem in die Herzhöhle hinabhängenden Klappenrande ist deutlich zu sehen, wie die innere bindegewebige Herzhülle (Intima) in das Perimysium der Aussenfläche des Organs übergeht (Taf. I, Fig. 2 end).

In der Mittellinie der Dorsalfläche des Herzens verläuft der sympathische Nerv, welcher nach Police (1903) mit zwei Wurzeln von der hinteren Convexität der suprapharyngealen Nervenmasse anfängt. Er liegt der longitudinalen faserigen Herzhülle an und enthält auf seiner Ausdehnung einzelne Nervenzellen, die möglicherweise autonome Herzzentren darstellen (Taf. I, Fig. 2 nzs).

Die Arterien beginnen am Herzen mit besonderen metamer angeordneten paarigen Klappen, die auf seiner ventralen Fläche gelegen sind. GaDSIKIEwICZ gibt eine wahrheitsgetreue, jedoch zu kleine Abbildung der Klappen beim Euscorpius (1. c., Taf. I, Fig. 9). Die arteriellen Klappen des Herzens bestehen fast ausschliesslich aus Muskulatur, die hier in Form einer verdickten, gleichsam aufgequollenen Schicht auftritt. Diese Klappen, zumindest diejenigen der Seitenarterien werden aus der Ringsmuskulatur des Herzens gebildet (Gadsikiewicz, 1. c., S. 9).

Diese knappe Schilderung muss man in manchen Beziehungen abändern und ergänzen. Der basale Teil der Arterie bläht sich in Form einer Zwiebel auf (Taf. I, Fig. 3 bar) und wächst mit der Aussenwand des Herzens zusammen. In die Höhle der Zwiebel hängt von den Rändern der Arterienmündung die Klappe hinab (Taf. I, Fig. $3 e a$ ); die Klappe zeigt auf dem Längsschnitte die Form eines zylindrischen Muffs, dessen distales Ende sich durch ein spaltähnliches Loch in das Arterienlumen öffnet (das Pfeilchen links auf Fig. 3, Taf. I).

Die Wand der Klappe ist räumlich mit der muscularis des Herzens verbunden, besteht jedoch nicht aus denselben Muskelfasern wie das Herz. Die typischen halbringförmigen Muskelfasern des Herzens sind am proximalen Ende der Klappenöffnung nicht mehr vorhanden (das reclite Pfeilchen auf Fig. 3, Taf. I). Die Wandung der Klappe ist an der Basis dünn, die des hinabhängenden Klappenteils verdickt. Ihre muscularis besteht aus dünnen, mit Fortsätzen versehenen, miteinander anastomosierenden Zellen, welche von allen Seiten von bindegewebigen Hüllen umkleidet sind; die letzten sind unmittelbar mit der Intima (Perimysium) des Herzens verbunden. Durch die Öffnung der Klappe verbindet sich die Intima derselben mit der äusseren bindegewebigen Hülle (Adventitia), die wiederum an der Klappenbasis in die Arterienintima übergeht (Taf. I, Fig. 3).

Die Hüllen der Muskelfasern sind durch interstitielles Bindegewebe mit 


\section{ANATOMIE DES BLUTGEFÄSSYSTEMS DER SKORPIONEN ${ }^{467}$}

grossen, bläschenähnlichen Kernen gebildet. Die Arterienwand ist räumlich mit dem Endokard verbunden. Sie besteht aus mit Fortsätzen versehenen Muskelzellen, welche sich von den glatten Muskelfasern der äusseren Herzhülle durch das Vorhandensein einer Querstreifung unterscheiden. Die Zellen sind ebenfalls von einem membranähnlichen Bindegewebe umkleidet, welches mit dem Stroma des Herzens verbunden ist. Die Arbeit der Klappe ist klar. Sie öffnet sich bei der Herzsystole, bei der Diastole drückt das zurückströmende Blut auf den hinabhängenden Teil der Klappe, wodurch dieselbe zugeschlagen wird. Zum Klappenschluss kann auch die Kontraktion der aufgeblähten Arterienbasis beitragen, wodurch das Blut in die Gefässe weitergetrieben wird.

GadsıkıEwicz findet, dass ,die arteriellen Wände aus äusseren Längsfasern und Ringsmuskulatur besteht" (1. c., S. 9). Die Längsfasern sind, höchstwahrscheinlich, ähnlich wie im Herzen bindegewebiger Natur. Die zirkuläre Muskulatur ,bildet nicht eine kompakte Schicht; die kontraktile Substanz der Fasern steht auf dem ganzen Umkreise vom Sarkolemm ab... Die Ringsmuskelfasern sind in den Arterien ganz glatt" (1. c., S. 9). Diese Beschreibung des Baues der Arterien ist nicht ganz richtig. Vor allen Dingen kann man nicht in den Arterien deutlich eine äussere Schicht unterscheiden, welche dem Ektokard des Herzens voll und ganz entsprechen würde. Der Grundbestandteil der Gefässwand ist die muscularis, welche aus sternförmigen sich miteinander verbindenden quergestreiften Muskelfasern besteht, welche aber keine kompakte Schicht bilden (Taf. I, Figg. 9, 7, 8 ema). Ihre Querstreifung ist sehr deutlich auf dem Präparate zu sehen; mit Giemsa färbt sich dieselbe sehr schwach, weshalb sie auf der Fig. 9 auch nicht dargestellt ist. Um die Querstreifung in schärfster Weise hervortreten zu lassen, muss Eisenhämatoxylin verwendet werden.

Dic Muskelfasern sind von membranähnlichen bindegewebigen Elementen überzogen, die Gadsikiewicz für ein von den Fasern abgelöstes Sarkolemm ansprach. Diese Ansicht ist irrig, da die geschilderten Gebilde zum Perimysium - zum Bindegewebe gerechnet werden müssen und keineswegs Hüllen darstellen, die sich selbst von den Fasern ablösten. Die Arterien (von der a. supraneuralis abgesehen) sind von innen ziemlich scharf konturiert dank dem Vorhandensein von einer homogenen, kernlosen bindegewebigen Platte, die besonders deutlich an tangentiellen Schnitten durch die innere Fläche des Gefässes zum Vorschein kommt (Taf. I, Fig. 9 end).

Diese soeben erwähnte Platte bildet die Intima des Gefässes und ist gewöhnlich in der Längsrichtung gefaltet. Die geschilderte Arterienschicht muss zur Kategorie der Grenzgebilde, wie sie Livanoff (I9I4) beschrieben hatte, gerechnet werden. Ein eigentliches Endothel, wie es Schneider im Jahre 1892 schilderte, scheint es in den Gefässen des Skorpions nicht zu geben.

Die Muskelfasern sind auch von aussen mit einem Bindegewebe um- 
kleidet, das sich unmittelbar in Lamellen fortsetzt, zwischen welche Nephrozyten eingelegt sind (Taf. I, Figg. 7, 9 neph). Demnach ist in den Arterien, ähnlich wie im Herzen, ein bindegewebiges Stroma vorhanden, in welchem eine Schicht sternförmiger quergestreifter Muskelfasern gelegen ist. Von innen ist das Gefäss mit einer homogenen kompakten Grenzplatte ausgekleidet, welche die Undurchdringlichkeit der Gefässwandung bedingt. In der Gefässwand können also nur mit Vorbehalt zur Bequemlichkeit der Schilderung eine tunica intima, tunica media und tunica adventitia unterschieden werden. Die kleinen Blutgefässe unterscheiden sich von den grösseren nur durch ihren Durchmesser.

In dem supraneuralen Gefässe, welches auf sich die Lymphdrüse trägt, scheint diese homogene Auskleidung zu fehlen,. die Intima ist hier oft in Schichten gespalten und wird dann für Blut- und Plasmaelemente leicht durchdringbar (PAwlowsky I9I5, 19I6). Die feinen Arterienäste öffnen sich bei den Skorpionen in zwischen den Organen vorhandene Lakunen. R. LANKester schreibt im Jahre 1885, dass ,these may be called, as in the custom, sinuses or lacunae; but they are truly veins with their own proper walls, though of non cylindrical form in cross section" (1. c., S. 374). Auf Fig. IO, Taf. 79 bringt BECK (1885) den Querschnitt eines perikardioventralen Muskels mit einem Gefässlumen innen. Houssay (I887), der Karminglyceringelatine injizierte, stellte das Vorhandensein von Lakunen fest, denen eigene Wandungen fehlten (Cephalothoracale und Nervenkettenlakune).

Beim Studium der Schnitte durch verschiedene Körperteile des Skorpions (Scorpio maurus, Buthus eupens, B. caucasicus, B. australis, Euscorpius usw.) können wir uns überzeugen, dass es Lakunensysteme zweierlei Natur gibt. So können sich die Arterien in Spalträume zwischen den Organen und in solche zwischen den Teilen der Organe öffnen, wobei die Oberflächen der Organe zugleich die Wände der Lakunen darstellen. Physiologisch entspricht dieser Teil des Blutgefässsystems, wo die Atmung der Körperzellen stattfindet, den Kapillaren der Vertebrata. Von hier gelangt das Blut in die mit eigrenen Wänden versehenen venösen Gefässe, deren Lage durch die früheren Autoren (NEwPORT, Blanchard) bereits klargestellt worden ist. Für diese Sinusse trifft die oben gebrachte Meinung Lankester's, dass sie echte Venen seien, vollständig zu. Dasselbe kann man auch von den pulmoperikardialen Gefässen sagen, welche das sauerstoffreiche Blut aus den Lungen in die Perikardhöhle leiten. Demnach müssen wir in unseren weiteren Ausführungen einfache Lakunen - Analogen der Kapillargefässe - von venösen Sinussen oder Kanälen unterscheiden.

Der mikroskopische Bau der venösen Kanäle ist sehr einfach. Von innen sind sie mit einem plattenähnlichen Bindegewebe ausgekleidet (Taf. I, Fig. Io end), in dessen Masse stellenweise auch Muskeln $(m)$ liegen können. In 
letztem Falle kann die Rede von allen drei Bestandteilen der Gefässwand sein: von einer aus Perimysium bestehenden Intima, von einer muscularis, welche aus geradlinigen quergestreiften Muskelfasern mit zwischen ihnen eingelegtem Endomysium gebildet ist und schliesslich von einer Adventitia, die durch die oberflächlichen Schichten des Perimysiums dargestellt wird. Es kommt häufig vor, dass die Wand des venösen Kanals nicht auf dem ganzen Umkreise gleich gebaut ist. So enthalten die pulmoperikardialen Venen eine muscularis nur in dem oberen, unteren und hinteren (auf die normale Körperlage des Skorpions bezogen) Teilen, während die vordere Wand lediglich aus plattenähnlichem Bindegewebe besteht, das eine unmittelbare Fortsetzung des Perimysiums darstellt. In derartigen Stellen des Gefässes ist selbst eine vorbehaltliche Einteilung der Wand in drei Schichten nicht angebracht.

Im allgemeinen kann man auch hinsichtlich der Venen sagen, dass dieselben aus bindegewebigen plattenähnlichen Gebilden bestehen, in deren Masse Muskelfasern derselben Art wie in den willkürlichen Muskeln des Skorpions lagern können.

Der Perikardbeutel ist von innen durch plattenähnliches Bindegewebe abgegrenzt, in welchem beim Färben mit Eisenhämatoxylin deutliche faserige Struktur hervortritt (Taf. I, Fig. 6 fin). Zwischen den Schichten sind runde oder ovale Kerne mit zahlreichen Chromatingranulis gelegen. Diese Kerne scheinen dieselbe Bedeutung wie diejenigen des Peri-, des Endomysiums, des plattenähnlichen Bindegewebes zwischen den Nephrozyten, des Stromas der Lymphknoten, des Herzens, der Blutgefässe usw. zu haben. Sie gehören, wie mir scheint, zu den indifferenten Elementen des Bindegewebes. Die Perikardwand färbt sich nach MALlory blau. Sie ist nur von unten und von den Seiten ausgeprägt, wo sie unmittelbar in die plattenähnlichen Elemente der Pulmoperikardialvenen, in die adventitia der Basis der unteren lateralen Arterien (Taf. I, Fig. 3 pro) und in das Perimysium der venoperikardialen -Muskelı des Herzens übergeht. Von der dorsalen Seite ist die Perikardwand durch die basale Platte der Hypodermis dargestellt, mit welcher auch die Anhängevorrichtung des Herzens verbunden ist. Hierzu muss noch bemerkt werden, dass in den coelothelialen Membranen mancher Würmer elastische Fasern beschrieben worden sind (LivanofF 19I4, I9I7).

Die von mir gebrachten Daten über den mikroskopischen Bau des Blutgefässystems cler Skorpione können mit den Resultaten der Untersuchung der Gefässe bei den Anneliden verglichen werden. Der allgemeine Plan ihrer Struktur ist ein gleicher. So ist in den Gefässen der Blutegel, nach LivanofF, „die innere Schicht... stets durch eine Grenzmembran dargestellt... Die äussere Schicht ist aus einem Teile des Körpermesenchyms mit seiner inneren Grenzmembran gebildet. Die mittlere Schicht ist aus zirkulären Muskeln im Rückengefäss gebildet, in den transversalen und abdominalen Gefässen augen- 
scheinlich aus ihren Homologen, aus den Zellen der eigentlichen mittleren Schicht" (1916, S. I88).

Im Vorderteil des Rückengefässes bei der Acanthobdella beschrieb Livanoff ein Endothel. Die Nachuntersuchung des entsprechenden Materials ergab, dass ein solches nicht bestehe: „die innere Auskleidung besteht überall aus einer typischen Grenzmembran, stellenweise nur spaltet sich (1. c., S. I83) dieselbe und ihrer inneren Lamina liegen dann Zellen an, welche ein Endothel vorspiegeln". Ihre Natur wird näher nicht bestimmt. Bei den Protoclepsis, Glossosyphonia und Pontobdella sind Zellen in der Intima des Rückengefässes kein einziges Mal gefunden worden; die Ringsmuskelzellen sind von allen Seiten in Hüllen aus Grenzgebilden eingeschlossen.

Selensky (19I5) weist in ganz bestimmter Weise darauf hin, dass bei den Ichthyobdellidae ein Endothel in den Gefässen nicht vorhanden ist. Ihre Intima ist "durch eine bindegewebige Membran, in welcher auch Zellelemente vorkommen können, dargestellt" (1. c., S. 178). Die bindegewebige Natur der Intima der Blutgefässe haben schon früher Leydig (1857), Fernandez (1905) und viele andere anerkannt. Der Letztgenannte gelangte zum Schluss, dass das Blutgefässsystem sämtlicher Coelomata aus einem primären Hauptbestandteile mesenchymalen Ursprungs und aus einem sekundären propulsatorischen Teile, welcher sich aus der Coelomwand entwickelt, bestehe. Auf Kosten des Mesenchyms entstehen die innere Hülle der Gefässe mit den ihr anliegenden Zellen des Endothels oder Pseudoendothels, die Blutzellen, Klappen usw.

Livanoff sowie auch Fernandez „sehen in dem Blutgefässystem im allgemeinen ein System trophocoeler Höhlen im Gewebe der Grundsubstanz" (1916, S. I9I). Seiner Meinung nach sei der Hauptbestandteil der Gefässwände lediglich die Adventitia mit ihrer Grenzmembran (Intima), mit anderen Worten nur das Mesenchym des Körpers der Hirudineen allein, während die übrigen Gebilde untergeordnete akzessorische Teile darstellen (1. c., S. 190-19I). Eine ähnliche Auffassung kann man auch auf die Skorpione ausdehnen. Als gemeinsames Element sämtlicher Teile des Blutgefässystems - des Herzens, der Arterien und der Venensinusse - tritt das interstitielle Bindegewebe auf, zwischen dessen Schichten folgende Elemente eingeschlossen sind: im Herzen - halbringförmige Muskelfasern, in den Arterien - sternförmige quergestreifte Zellen und in den Venen - Muskelfasern mit parallelen Rändern. Man kann jedoch nicht von einem Prävalieren des interstitiellen Bindegewebes gegenüber den Muskelzellen sprechen, da nur wenige Abschnitte der Venen ausschliesslich aus Bindegewebe bestehen.

Die von Sснімкеwitsch bei den Pedipalpiden (I906) entdeckten Kardiocoelomöffnungen fehlen bei den Skorpionen. Ihnen homolog sind die

' Wie das im Herzen des Skorpions der Fall ist. 


\section{ANATOMIE DES BLUTGEFÄSSYSTEMS DER SKORPIONEN ${ }^{471}$}

arteriellen Öffnungen des Herzens, welche mit zwiebelähnlichen Erweiterungen der Arterien (ventralen) bedeckt sind (Taf. I, Fig. 3 ea). SснімкеwITSCH wies auf die Ähnlichkeit zwischen denselben bei den Pedipalpiden und Orthoptheren ${ }^{1}$ hin. Bei den letzten sind sie von Kowalewsky gefunden worden ( 1894 ). Eine derartige Zusammenstellung gibt uns die Berechtigung, von einer Homologie der arteriellen Öffnungen des Skorpions und der Kardiocoelomöffnungen der Orthopthera zu sprechen, bei welchen die genannten Gebilde eben Reste ehemals vorhandener Blutgefässe darstellen. Augenfällig ist auch die Ähnlichkeit im mikroskopischen Bau der arteriellen Klappe des Skorpions und dem der Ostialklappen der Äschnalarve (von ZAVARZIN im Jahre I9I I beschrieben). Diese Organe sind aus einem retikulären Gebilde gebildet, das durch Spaltung der bindegewebigen Herzadventitia entstanden ist. In den Maschen des Bindegewebes liegen zahlreiche Leukozyten. Zur volleren Ähnlichkeit mit den Skorpionen fehlt noch das Vorhandensein verzweigter Muskelfasern.

Es ist sehr schwierig, vorläufig einen detaillierten Vergleich des mikroskopischen Baues des Skorpionherzens mit dem anderer Arachnoida durchzuführen, weil die Forscher noch nicht genügend den Entwicklungsgrad des Sarkolemms der Muskelfasern und des Perimysiums derselben klargestellt haben. TARnan I (1904) weist zum Beispiel darauf hin, dass beim Thelyphonus das Herz innen mit einer Intima ausgekleidet ist; für eine Intima hielt er nämlich das von den Muskeln abstehende Sarkolemm, jedoch letzten Endes gelangt er zur Überzeugung, dass es in der Tat keine Intima gebe. SснімкEWITSCH (rgo6) schildert auf Grund seiner Beobachtungen und derjenigen von Franz (I904) den Bau des Herzens beim Thelyphonus folgendermassen: es besteht aus denselben Schichten wie das Herz der Spinnen, und zwar aus einer bindegewebigen Adventitia, einer dünnen Schicht longitudinaler Muskelfasern und einer mächtigen Lage zirkulärer Fasern. Nach Loman (Ig03) gibt es im Herzen der Phalangidae äussere Längsmuskelfasern und innere Ringsmuskelfasern; dieser Autor nimmt auch an, dass es eine äussere und innere sehr zarte Hüllen gebe.

Bei der Galeodes (I896) sieht Bernard innen im Herzen eine „fine refractive membrane". GadsikiEwicz hält sie für ein Sarkolemm der Muskelfasern, welches auch bei den anderen von ihm untersuchten Arachnoide einen Anteil an der Bildung der homogenen Intima nehme. Nach Zavarzin (I9II) sind bei.der Äschnalarve die Múskelelemente des Herzens von aussen mir einer elastische Fasern enthaltenden Adventitia, von innen mit einer Intima bekleidet. Die Adventitia ist von der Seite, welche den Muskelzellen anliegt, mit einer Grenzmembran bedeckt, die sich mit der Intima vermittelst einer zwischen die medialen Zellgrenzen eintretenden Nahtquerleiste verbindet. Die

${ }^{1}$ Peskrer (1906) hält die Seitenarterien der Arachnoida für modifizierte Kardiocoelomöffnungen. 
Muskelzellen sind also von allen Seiten von diesen Gebilden umgeben, welche nach der Annahme Zavarzin's Ablömmlinge des Sarkolemms darstellen. Sollten wir diese Gebilde für Grenzgebilde oder Perimysium ansprechen, so würden wir eine wesentliche Ähnlichkeit in den Details des mikroskopischen Baues des Herzens beim Skorpion und der Äschnalarve erhalten, wobei die elastischen Fasern der Adventitia bei der Äschna den ,glatten” Muskelfasern derselben Schicht im Skorpionherzen entsprechen werden. In gleicher Weise könnte man auch einen detaillierten Vergleich mit den Blutgefässen der Anneliden vornehmen. Nachdem nun eine Ähnlichkeit im Bau des Herzens bei den Würmern, dem Skorpion und der Äschnalarve festgestellt worden ist, so ist erst recht zu erwarten, dass neuere Untersuchungen an Herzen anderer Arachnoida Tatsachen ans Tageslicht fördern werden, die die wichtige Anteilnahme nicht nur der Muskelelemente, sondern auch des Bindegewebes an der Bildung der Herz- resp. Gefässwand bestätigen werden.

Die trophocoele Theorie von LANG (I903) sieht nunmehr, wie SELENSKY mit Recht bemerkt (I9I5), einer Abänderung entgegen, da auf die wesentliche Rolle des Bindegewebes in der Bildung der Gefässwände im allgemeinen und der Intima im besonderen hingewiesen wird. Freilich hat auch LaNG bemerkt, dass das Bindegewebe bei den Mollusca und Tunicata an der Bildung der Blutgefässe beteiligt sei; die letzten stellen schisocoele Kanäle zwischen der Organen, in der Muskulatur und im Bindegewebe dar, welches eben die Gefässwände bilde, und nur das Herz sei der einzige mit eigenen Muskelwänden versehene Bestandteil des Blutgefässsystems.

\section{ERKLÄRUNG DER ABBILDUNGEN.}

$$
\text { Bedeutung der Buchstaben: }
$$

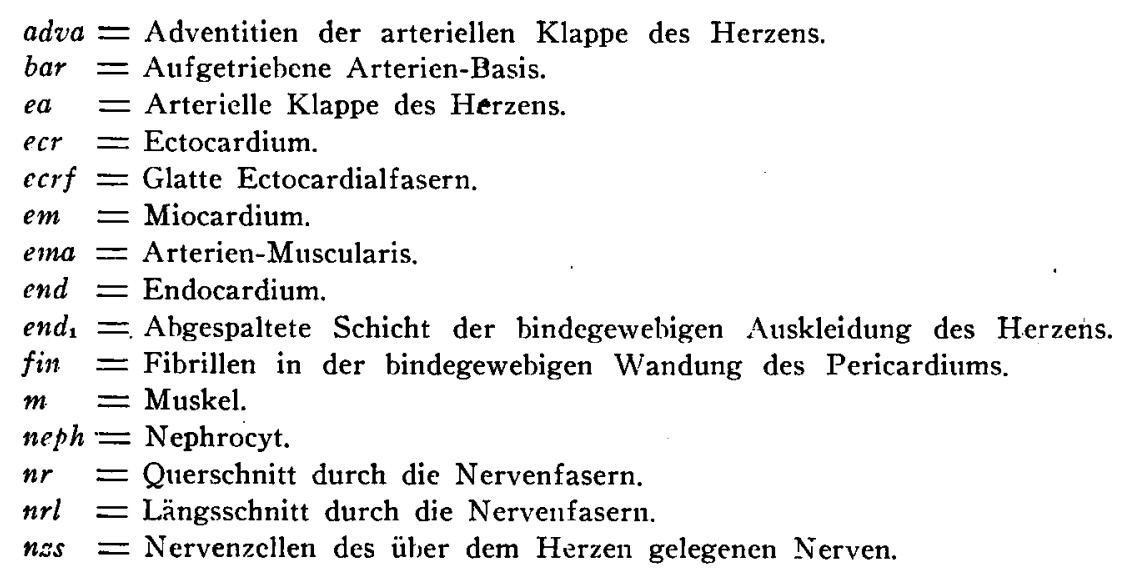




\section{ANATOMIE: DES BLUTGEFÄSSYSTEMS DER SKORPIONEN ${ }^{473}$}

\section{TAFEL $\mathrm{I}$.}

Fig. I. Buthus australis L. Teil des Längsschnittes durch das Herz. Die innere Auskleidung ist stellenweise gespalten und steht ab (end). Eosin-Azur. Z. 1/12 Obj. hom. imm. oc. o.

Fig. 2. Buthus eupens L. Längsschnitt durch das Herzostium. Aus dem Ostium ergiesst sich in die Herzhöhle Blut. Heisses Sublimat mit Essigsäure. Giemsa Z. ob. DD; oc. 2.

Fig. 3. Buthus australis L. Längsschnitt durch die Stelle, wo die ventrale Arterie vom Herzen abgeht. Die Arterie bildet an ihrer Basis eine zwiebelartige Erweiterung (bar), in welcher sich die Muffklappe (dieselbe ist mit doppelten Pfeilchen bezeichnet) befindet. Eosin-Azur. Z. Obj. ${ }^{1 / 12}$ hom. imm. ce. 4.

Fig. 4. Buthus australis L. Teil eines Längsschnittes in der Medianlinie der Dorsalseite des Herzens. Es ist ein Teil des suprakardialen Nerven $(n r l)$ mit Nervenzellen $(n z s)$ im Verlauf der Fasern zu sehen.

Fig. 5. Buthus australis L. Eine Nephrozytengruppe an der Aussenfläche des Herzens. Zwischen den Nephrozyten verlaufen homogene, von interstitiellem Bindegewebe umgebene Fasern der äusseren Herzhülle. Eisenhämatoxylin (HeidenHein). Lichtgrün. Z. Obj. $1 / 12$ hom, imm. oc. I.

Fig. 6. Buthus australicus $L$. Teil der Wandung des Perikardbeutels mit in der Tieie der Wand lagernden Fibrillen (fin). Dieselbe Färbung wie am vorangegangenen Präparat. Z. Obj. $1 / 12$ hom. imm. oc. 4.

Fig. 7. Scorpio mantus L. Querschnitt durch eine Arterie, die einen der grossen Nerven der subpharyngealen Ganglienmasse begleitet. Zenker-Formol-Osmium. Eienhämatoxylin (HeIdenhfils). Z. Obj. 1/12 hom. imm. oc. I.

Fig. 8. Buthus eupens L. Querschnitt durch eine die Leber durchbohrende Arterie. Heisses Sublimat mit Essigsäure. Mann-Hollande. Z. Obj. 1/12 hom. imm. oc. I.

Fig. 9. Scorpio naurus L. Tangentieller Schnitt durch die Arterie, an dem die innere Auskleidung und Intima zu sehen sind. Starke Vergrösserung.

Fig. ro. Buthus cupens L. Teil eines Schnittes durch die Wand eines Sinus, der den Lungensinus mit der Perikardhöhle verhindet. CıRNoY. Giemsa Z. Obj. 1/12 hom. imin. oc. I.

\section{LITERATURVERZEICHNIS.}

Bernard. The ccmrarativ Morpholcgy of the Galecdidae. Trans. Linn. Soc. London. Vol. 6. 1896.

Bi.ANChaRD, E. L'organisation du régne animal. Paris. I85I-I859.

Durour, L. Histoire anatomique et physiologique des Scorpions, Mém. prés. pour divers savants à l'Acad. Sciences. Vol. 14. 1856.

Duvernoy. Fragments sur les organes de genération de divers animaux. Mém. Acad. Sc. Inst. France. Vol. XXIII. I853.

GnuSıKIE.WICZ, W. Zur Phylogenie des Blutgefässystems bei Arthropoden. Zool. Anz. V. 29. 1905.

- Zur Histolcgie des Blutgefässystems bei Arachnoiden Mém. de l'Académ. des Sciences de St.-Pétersbourg. VIII-e sér. Classe physico-mathćm. Vol. XXII, Nr. 7. 1908. (Russisch.)

Ftrnandez. Zur mikroskop. Anatomie des Blutgefässystems der Tunicaten. Jenaische Ztschr. f. Naturwiss. Vol. XXXIX. NF. XXXII. Bd. 
Franz. Oher die Struktur des Herzens und die Entstchung der Blutzellen bei Spinnen. Zcol. Anz. Vol. XXVII. Ig04.

Houssıy, F. Note sur le système artèriel des Scorpions. C. R. A. Sc. Vol. 103. I886.

- Sur la lacune sanguine périncrveuse, dite artère spinale chez les scorpions, et sur l'organe glandulaire annexe. Ibidem. Vol. I04. I887.

Kowalewsky, A. Etudes expérimentales sur le cœur de quelques Orthoptères. Arch. Zool. Expér. I\&94.

Kowalewsky, A. und Schulgin, M. Zur Entwicklungsgeschichte des Skorpions (Androctonus ornatus). Biol. Ctl. Vol. 6. I8s9.

LanG, A. Beiträge zu einer Trophocöltheorie. Jena. 1903.

Livanofr. Grenzgebilde der Polychaeta und die allgemeine morphologische Bedeutung dieser Gebilde. Arbeiten der Naturforscher-Gesellschaft an der Universität in Kasan. Vol. XLVI. I9I4. (Russisch.)

Livanorf et Bourorf. Etudes histelcgiques sur les système vasculaire chez les Hirudinées. Journ. de la Section Zoologique de la Soc. des Amis des Sciences Naturelles. Nouv. Sér. Vol. 1II. No. 4. 1916.

Lomann, J. Vergleichend anatomische Untersuchungen an chilenischen und anderen Opilinoiden. Zool. Jahrb. Abt. Anat. Vol. 3. Suppl. 6. I903.

New poRT. Nervous and Circulatory Systems in Myriopoda and Macrourous Arachnida. Philos. Transact. of the Royal Scciety. Part 1I. 1843.

Pawlowsky, E. Uber die phagocytären Organe und die Phagocytose beim Scorpio maurus. Arbeiten des Landwirtschaftlichen Bakteriologischen Laboratoriums. Bd. VI. I916. (Russisch.)

Pesker, D.; in Zool. Anz. Vol. 34. 1908.

Petrunkewitsch, A. The circulatory System and Segmentation in Arachnida. Journ. of Morphology. Vol. 36. No. 2. I922.

Ray Lankester, Bfhnam, W., and Miss Beck. On the Muscular and Endoskeletal Systems of Limulus and Scorpio, with Sone Notes on the Anatomy and Generic Characters of Scorpions. Trans. Zool. Lond. Vol. XI. 1885.

TARNani. Zur Anatomie von Tclyphonus caudatus. 1904.

Schimkewitsch, W. Uther die Entwicklung von Telyphonus caudatus, vergleichend mit derjenigen einiger anderer Arachniden. Ztschr. f. wiss. Zool. Vol. 8I. Ig06.

Schneiner, A. Mélanges arachnologiques. Tablettes Zoolog. Poitiers. Vol. 2. I892.

Selensky, V. Studien über die Morphologic und Systematik der Hirudinen. I. Organisation der Ichthyobdelliden. St. Petersburg I9I5. (Russisch.)

Zawarzın, A. Histologische Studien über Insekten. I. Das Herz der Äschnalarven. Zeit. Wiss. Zool. Vol. XCVII. I9II. 


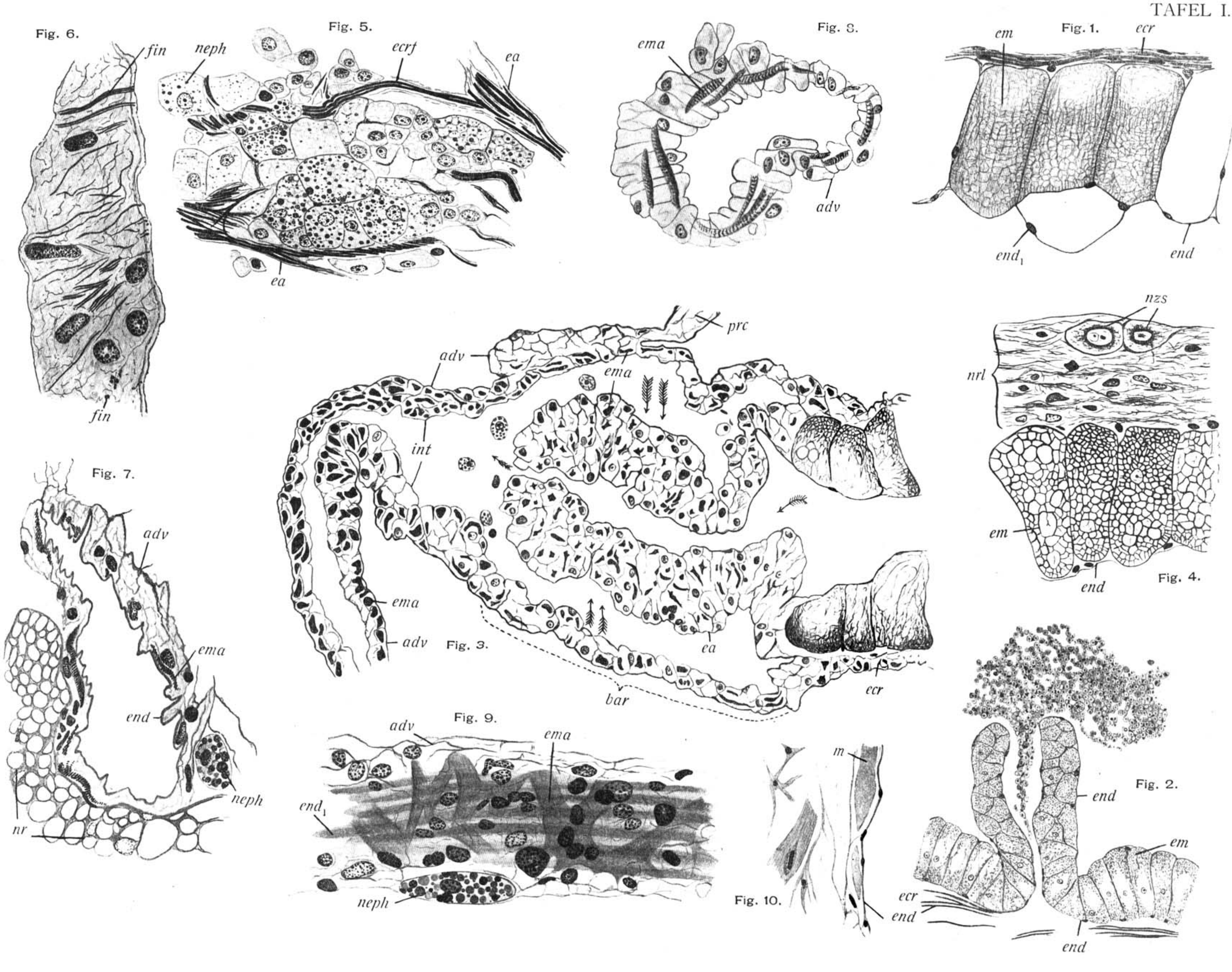

